\title{
Surveillance of the Naural Foci of Especially Dangerous Infections in Southern Ukraine
}

\author{
Zoya Nekhoroshykh*, G.M. Dzhurtubayeva, N.M. Protsyshyna, N.V. Pilipenko, S.V. \\ Pozdnyakov, N.A. Popova and E.A. Egorova
}

Mechnikov I.I. Ukrainian Research Antiplague Institute of the Ministry of Health of Ukraine, Odessa, Ukraine

\section{Objective}

Comprehensive ecological, epidemiological and microbiological investigations of natural foci of EDI in southern Ukraine were carried out

\section{Introduction}

Surveillance of the natural foci of Especially Dangerous Infections (EDI) is necessary due to their etiological, epidemiological, and clinical diversity, their global spread and overall negative impact on public health. Some EDI pathogens with natural foci are also potential agents for biological weapons. Study of the EDI characteristics is important for development of an effective epidemiological protection system. Francisella tularensis is one of the most virulent human microorganisms and a critical Category A biological agent. In Ukraine, tularemia natural foci are registered in 23 of the 25 regions. We conducted integrated ecological-epidemiological and microbiological research on the EDI natural foci for the past 20 years.

\section{Methods}

We studied ecological-epidemiological data (using retrospective analyses) and isolated $F$. tularensis strains by molecular-genetic (PCR, VNTR-Analysis), bacteriological, immunological, environmentalepidemiological, statistic methods.

\section{Results}

We detected favorable conditions for formation and long-term functioning of EDI natural foci of various etiologies in ecosystems of southern region of Ukrainian. Here tularemia natural foci were registered in Kherson and Odessa regions, and the largest outbreak of tularemia in humans in Ukraine occurred - 100 people fell ill in 1998 in the Odessa and Mykolaiv regions. The sources of infection were small mammals (forest and field mice), and hare. In our serological study of tularemia vectors, positive field data results confirmed the stability of tularemia natural foci. Seventeen strains of $F$. tularensis subsp. holarctica were isolated from different sources (rabbits, rodents, ticks, water). We conducted VNTR-analysis of the natural isolates, identified 9 genotypes, and constructed genetic passports of individual $F$. tularensis strains. In some Ukrainian southern regions, habitats containing natural foci revealed circulation of various EDI pathogens (tularemia, psittacosis, leptospirosis, arboviruses), which requires a polynozological approach to their monitoring, considering the overlap of vectors.

\section{Conclusions}

Eco-epidemiological and microbiological research in southern Ukraine have established the widespread presence of EDI of various etiologies. The presence of overlapping natural foci indicates that the southern region is an area with high risk of epizootic and epidemic complications. Use the molecular genetic research methods promote a science-based system for confirming areas containing EDI-specific territories and optimize the potential for prevention of infections near natural foci.

\section{Keywords}

EDI natural foci; strains genotyping; diagnosis; monitoring; prevention

\author{
*Zoya Nekhoroshykh \\ E-mail:nm_pri@mail.ru
}

